\title{
ОЦЕНКА ЭФФЕКТИВНОСТИ ЛЕЧЕНИЯ ХРОНИЧЕСКОГО ЭНДОМЕТРИТА У ПАЦИЕНТОК ПРИ ПОДГОТОВКЕ К ВСПОМОГАТЕЛЬНЫМ РЕПРОДУКТИВНЫМ ТЕХНОЛОГИЯМ
}

\begin{abstract}
EVALUATION OF THE EFFECTIVENESS
OF THE TREATMENT OF CHRONIC
ENDOMETRITIS IN PATIENTS IN
PREPARATION FOR ASSISTED
REPRODUCTIVE TECHNOLOGIES
E. Kosykh

I. Korotkikh

A. Filin

Summary. The article presents generalized data of foreign and domestic literature on inflammatory changes in the endometrium and their role in the formation of infertility in women of reproductive age. It was noted that chronic endometritis (CE) is characterized by a fairly high resistance of the flora to drug therapy and an increase in the duration of treatment, which confirms the relevance of the search for effective and relatively inexpensive methods of treating $C E$ in preparation for assisted reproductive technology (ART) programs. The use of ART allows one to achieve the implantation of viable embryos into the uterine cavity exclusively under conditions of receptive endometrium, which makes it important to study the problems of an integrated approach to the treatment of CE using immunomodulating therapy. The results of this work indicate the advisability of administering the drug derinat to women suffering from CE.
\end{abstract}

Keywords: chronic endometritis, infertility, miscarriage, endometrium, in vitro fertilization (IVF).

\section{Актуа^ьность}

U ирокомасштабное применение у женщин в активном детородном возрасте внутриматочных манипуляций (гистероскопий, абортов, ВМС и проч.) привело к тому, что хронический эндометрит (ХЭ) как нозология приобрел актуальность, за счет повышения частоты этого заболевания в последние несколько лет $[1,2,3,4,5]$. Хронический эндометрит является клинически значимой патологией с позиции репродукции и требует обязательной диагностики и лечения у женщин, страдающих бесплодием, при использовании про-
Косых Екатерина Владимировна

Аспирант, ФГБОУ ВО Воронежский Государственный Медицинский Университет им. Н. Н. Бурденко ekaterina1991kosykh@gmail.com Коротких Ирина Николаевна

Д.м.н., профессор, ФГБОУ ВО Воронежский Государственный Медицинский Университет

им. Н. Н. Бурденко

Филин Андрей Анатольевич

К.м.н., ФГБОУ ВО Воронежский Государственный Медицинский Университет им. Н.Н. Бурденко

Аннотация. В статье представлены обобщенные данные зарубежной и отечественной литературы о воспалительных изменениях в эндометрии и их роли в формировании бесплодия у женщин репродуктивного возраста. Отмечено, что хронический эндометрит (ХЭ) характеризуется довольно высокой резистентностью флоры к лекарственной терапии и увеличением сроков лечения, что подтверждает актуальность поиска эффективных и относительно недорогих способов лечения ХЭ при подготовке к программам вспомогательных репродуктивных технологий (ВРТ). Применение ВРТ позволяет достичь имплантации жизнеспособных эмбрионов в полость матки исключительно в условиях рецептивного эндометрия, что обуславливает актуальность изучения проблемы комплексного подхода к терапии ХЭ с применением иммуномодулирующей терапии. Результаты данной работы свидетельствуют о целесообразности назначения препарата деринат женщинам, страдающим ХЭ.

Ключевые слова: хронический эндометрит, бесплодие, невынашивание беременности, эндометрий, экстракорпоральное оплодотворение (ЭКО).

грамм вспомогательных репродуктивных технологий (BPT) $[1,6]$.

Вспомогательные репродуктивные технологии (ВРТ) -это методы преодоления бесплодия, при которых отдельные или все этапы зачатия и раннего развития эмбрионов осуществляются вне организма женщины.

Проведение вспомогательных репродуктивных технологий (ВРТ) как медицинских услуг осуществляется по медицинским показаниям в случае отсутствия противопоказаний и при наличии' письменного информиро- 
ванного согласия пациентов. При этом следует отметить тот факт, что лечение ХЭ является всего лишь подготовительным этапом к ВРТ, поскольку успешная имплантация жизнеспособных эмбрионов в программах экстракорпорального оплодотворения возможна только при наличии рецептивного эндометрия.

Значительное развитие современной фармакотерапии различных нозологий, в том числе и воспалительных заболеваний органов малого таза (ВЗОМТ) у женщин, не привело к резкому скачку эффективности их лечения. Современные особенности течения ХЭ обусловливают некоторые сложности ведения женщин с этой патологией, среди которых наиболее актуальными являются: полиэтиологичная изменчивость структуры ХЭ, трудности идентификации микробного агента в эндометрии, волнообразно-прогрессирующее течение данной патологии, ведущее к нарастающему во времени повреждению эндометрия; отсутствие самовосстановления эндометрия при длительности персистенции в нем повреждающего агента более 2 лет даже после элиминации последнего $[7,8]$.

Описанные особенности ХЭ затрудняют выбор этиотропного лечения и требуют проведения дополнительных терапевтических мероприятий при длительном течении заболевания с целью устранения каскада вторичных изменений эндометрия.

Учитывая воспалительную природу заболевания, целесообразность проведения антибактериальной терапии у пациенток с ХЭ в настоящее время не вызывает сомнений, однако до сих пор спорным остается вопрос о характере противомикробной терапии. Выявление же стерильных посевов из полости матки и цервикального канала при наличии клинических проявлений ХЭ наводят на мысль о том, что имеется вирусная инвазия в эндометрии, что в свою очередь является показанием для проведения иммуномодулирующей терапии. При хронических заболеваниях в очаге воспаления присутствуют и иммуносупрессорные макрофаги, предупреждающие разрушительные аутоиммунные повреждения.

«Первую линию» защиты от инфицирования представляют эффекторы врожденного иммунитета, которые обеспечивают своевременное выявление чужеродных инфекционных агентов и их быструю элиминацию. Среди сигнальных рецепторов врожденного иммунитета выделяют генетически детерминированные структуры TLRs. TLRs - это трансмембранные белки, играющие центральную роль в инициации врожденного иммунного ответа. TLRs формируют воспалительную реакцию, направленную на эрадикацию инфекционного агента. Открьггие TLRs в женском репродуктивном тракте обозначило их потенциальное клиническое значение.
TLRs играет важную роль в осуществлении противомикробной защиты эндометрия. Исходя из вышеперечисленного, возникает задача подбора терапевтического средства, сочетающего в себе свойства иммуномодулятора с выраженным репаративным эффектом. В данном аспекте интерес представляет отечественный препарат деринат. Иммуномодулирующий эффект, которого обусловлен взаимодействием действующего вещества препарата с патогенраспознающими рецепторами (TLRs), что в свою очередь стимулирует факторы роста эндотелиоцитов и образование новых сосудов.

В данный момент времени оправданным считается проведение морфологической диагностики ХЭ с целью предотвращения развития необратимых структурных изменений эндометрия и выбора тактики ведения пациентки (своевременное назначение противовоспалительной терапии).

Залогом успешного завершения вспомогательных репродуктивных технологии является предварительная комплексная оценка состояния женщины и проведение своевременной этиологически и патогенетически обоснованной терапии выявленной патологии эндометрия.

\section{Цель исслеАования}

Изучение клинической эффективности препарата Деринат ${ }^{\circledR}$ (раствор для внутримышечного введения 15 мг/ мл, флакон) в комплексной терапии ХЭ, ассоциированным с бесплодием у женщин репродуктивного возраста, заключающейся в повышении эффективности ВРТ.

\section{Задачи исследования}

1. Купирование клинических симптомов при их наличии (болей внизу живота тянущего и ноющего характера, болезненности, увеличения и уплотнения матки, положительная динамика по УЗИ: повышение однородности эхоструктуры эндометрия, рост эндометрия во 2 фазе менструального цикла);

2. Эффективное лечение инфекций, передающихся половым путем;

3. Уменьшение количества повторных внутриматочных вмешательств;

4. Восстановление репродуктивной функции.

\section{Материалы и метолы исследования}

Клиническая часть исследования проводилась на базе кафедры акушерства и гинекологии N1 ФГБОУ ВО «Воронежский государственный медицинский университет им. Бурденко» Министерства здравоохранения Российской Федерации. Лабораторные исследования проводились на базе клинико-диагностической лабо- 
Таблица 1. Алгоритм проведения врачебных визитов в исследовании

\begin{tabular}{|c|c|c|}
\hline Этап исследования & Срок исследования & Описание алгоритма действий \\
\hline 1-й визит & \multirow[t]{4}{*}{$\begin{array}{l}\text { 1-й месяц } \\
\text { наблюдения }\end{array}$} & $\begin{array}{l}\text { скрининг, на котором перед включением пациентов в исследование (0-й } \\
\text { день) } \\
\text { проводится обследование: изучение анамнеза (наличие хронического } \\
\text { эндометрита в анамнезе, аборты; } \\
\text { внутриматочные инвазивные манипуляции (выскабливание полости матки, } \\
\text { введение или извлечение ВМК); бесплодие; привычное невынашивание, } \\
\text { длительность заболевания, сведений о сопутствующих заболеваниях и } \\
\text { сопутствующей терапии), оценка общих } \\
\text { показателей (ЧСС, АД, температура тела), бимануальное исследование }\end{array}$ \\
\hline 2-й визит & & УзИ органов малого таза на 5-7дни менструального цикла \\
\hline 3-й визит & & $\begin{array}{l}\text { На 9-10 менструального цикла биопсия эндометрия (вакуум-аспирация } \\
\text { эндометрия мануальным вакуум-аспиратором Pentcraft) с последующим } \\
\text { микробиологическим (Фемофлор) и иммуногистохимическим (синдекан-1) } \\
\text { анализом состава полости матки, кофокусный анализ экспресси TLR9 }\end{array}$ \\
\hline 4-й визит & & УзИ органов малого таза на 22-24 день менструального цикла \\
\hline 5-й визит & $\begin{array}{l}\text { 2-й месяц } \\
\text { наблюдения }\end{array}$ & $\begin{array}{l}\text { применение терапии согласно глруппе больных с 1-го дня менструального } \\
\text { цикла }\end{array}$ \\
\hline 6-й визит & \multirow{3}{*}{$\begin{array}{l}\text { 3-й месяц } \\
\text { наблюдения }\end{array}$} & УзИ органов малого таза на 5-7 день менструальнго цикла \\
\hline 7-й визит & & $\begin{array}{l}\text { На 9-10 менструального цикла биопсия эндометрия (вакуум-аспирация } \\
\text { эндометрия мануальным вакуум-аспиратором Pentcraft) с последующим } \\
\text { микробиологическим (Фемофлор) и иммуногистохимическим (синдекан-1) } \\
\text { анализом состава полости матки, кофокусный анализ экспресси TLR9 }\end{array}$ \\
\hline 8-й визит & & УЗИ органов малого таза на 22-24 день менструального цикла \\
\hline 9-й визит & 4-6й месяцы наблюдения & 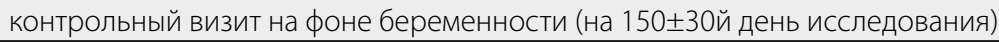 \\
\hline
\end{tabular}

ратории БУЗ ВО «Воронежская областная клиническая больница № 1». Патоморфологические исследования осуществлялись в отделении морфологических анализов ГУз «Воронежское областное патологоанатомическое бюро».

Проведенное исследование инициировалось держателем регистрационного удостоверения препарата деринат $^{\circledast}$ (000 «ФармПак», Россия) и соответствовало структуре пострегистрационного исследования эффективности по однородности выборок групп, возрасту пациентов, полу, частоте заболеваний за период исследования, фоновой патологии.

В исследование было включено 60 пациенток в возрасте от 20 до 41 года, что соответствовало критериям активного репродуктивного возраста с установленным диагнозом: привычное невынашивание беременности, хронический эндометрит. К критериям исключения пациенток из исследования относилось: индивидуальная непереносимость применяемой терапии; наличие заболеваний, требующих назначения антибактериальной, противовирусной и иммуномодулирующей терапии и/ или хронических заболеваний мочеполовой системы; участие в каких-либо клинических исследованиях в течение предыдущего месяца и/или отказ пациента принимать участие в исследовании. Все больные, включенные в исследование, получали лечение в условиях гинеколо- гических отделений (N1, N2 и N4) Перинатального центра «Воронежская областная клиническая больница № 1 ». На протяжении исследования проводилось 9 визитов пациенток (таблица 1). Период наблюдения составил 6 месяцев.

Исследовательские группы представлены следующим образом: группа 1 (основная) - 30 пациенток с привычным невынашиванием беременности и хроническим эндометритом в возрасте от 21 до 41 года, которым проводилось стандартное антибактериальное лечение в комплексе с применением препарат Деринат раствор для внутримышечного введения 15 мг/мл (флакон), производитель 000 «ФЗ Иммуннолекс», Россия) по 5 мл (75 мг) 1 раз в сутки через 48 часов в течение 10 дней, 10 инъекций на курс; группа 2 (контрольная) - 30 пациенток с привычным невынашиванием беременности и хроническим эндометритом в возрасте от 21 до 41 года, которым проводилось стандартное антибактериальное лечение без комплексного применения иммуномодулирующей терапии. Антибактериальные препараты применяются согласно результатам бактериологического исследования по утвержденным схемам лечения ЗППП (КП утвержденных Экспертной комиссией, протокол М 10 от 4 июля 2014 года).

Морфологические методы исследования включали в себя: биопсия эндометрия (вакуум-аспирация эндоме- 
Таблица 2. Соотношение клинических симптомов ХЭ в исследовании до начала лечения

\begin{tabular}{|l|l|l|}
\hline Клинический симптом ХЭ & $\begin{array}{l}\text { Группа1 } \\
\text { n(\%) }\end{array}$ & $\begin{array}{l}\text { Группа2 } \\
\text { n(\%) }\end{array}$ \\
\hline Нарушение менструального цикла & $(80 \%)$ & $(93 \%)$ \\
\hline Бесплодие (трубно-овариальные причины) & $(40 \%)$ & $(44 \%)$ \\
\hline Бесплодие (гормональное) & $(30 \%)$ & $(24 \%)$ \\
\hline $\begin{array}{l}\text { Воспалительные заболевания репродуктивной } \\
\text { системы в анамнезе }\end{array}$ & $(65 \%)$ & $(58 \%)$ \\
\hline Неудачные попытки ЭКо & $30(100 \%)$ & $30(100 \%)$ \\
\hline
\end{tabular}

Таблица 3. Соотношение иммунокомпетентных клеток у пациенток в исследовании

\begin{tabular}{|l|l|l|l|l|}
\multirow{2}{*}{ Maркеры } & \multicolumn{2}{l|l}{$\begin{array}{l}\text { Группа1 } \\
\text { (ср. кол-во кл/объем.плотн.) }\end{array}$} & \multicolumn{2}{l|}{$\begin{array}{l}\text { Группа2 } \\
\text { (ср. кол-во кл/объем.плотн.) }\end{array}$} \\
\cline { 2 - 5 } & До лечения & После лечения & До лечения & После лечения \\
\hline CD68 & $16,8 / 11,1$ & $14,9^{*} / 9,6^{*}$ & $16,8 / 10,6$ & $16,7^{*} / 10,5^{*}$ \\
\hline CD163 & $21,7 / 13,6$ & $21,4 / 14,1^{*}$ & $21,3 / 13,2$ & $19,2 / 11,4$ \\
\hline CD138 & $1,2 / 0,8$ & $1,5^{*} / 0,9^{*}$ & $1,3 / 0,8$ & $1,2^{*} / 0,8^{*}$ \\
\hline
\end{tabular}

Примечание; * $-p<0,001$

трия мануальным вакуум аспиратором Pentcraft; исследование биоценоза урогенитального тракта у женщин репродуктивного возраста методом ПЦР с детекцией результатов в режиме реального времени (Фемофлор); иммуногистохимический анализ (синдекан-1) биопсийного материала полости матки; кофокусный иммуногистохимический анализ экспрессии TLR9 с помощью моноклональных антител; кофокусный иммуногистохимический анализ М 1 или М2 фенотипа резидентных макрофагов с помощью моноклональных антител; кофокусный иммуногистохимический анализ PD1 с помощью моноклональных антител.

Оценка эффективности терапии на основании: восстановления репродуктивной функции (наступление-беременности, благополучное протекание беременности), прироста эндометрия по данным УЗИ.

Проведение статистической обработки результатов исследования осуществлялось с помощью пакетов программ Exce1 2010, Statistica 10.0, SPSS for Windows с использованием параметрических или не параметрических критериев (t-критерий Стьюдента, а также критерии Манна - Уитни и Вилкоксона соответственно). Статистически значимыми считались различия при $\mathrm{p}<0,05$.

\section{Характеристика \\ исслеАуемого препарата}

В исследовании оценивали клиническую эффективность препарата Деринат (раствор для внутримышечно- го введения 15 мг1мл, флакон) в комплексной терапии ХЭ, ассоциированным с бесплодием у женщин репродуктивного возраста. Препарат относится к фармакотерапевтической группе: иммуномодулирующие средства, регенерант, репарант.

Согласно литературным данным Деринат ${ }^{\circledR}$ обладает свойствами агониста агонистом толл-подобных рецепторов 9 (Toll-like reseptors 9, TLR9, CD289) Иммуномодулирующий эффект обусловлен взаимодействием действующего вещества препарата (цитозин-гуанин (CG)) с патоген-распознающими рецепторами (TLR9) на иммунокомпетентных клетках, что приводит к последующей активации целого ряда иммунных механизмов. Стимуляция TLR9 макрофагов, а также их IFNy, приводит к активации трёх уровней макрофагального ответа и повышению репаративного потенциала клеточных структур.

\section{Результаты \\ собственных исслеАований}

В результате проведенных исследований установлено, что в 100\% случаев как в первой, так и во второй группах исследования ведущим клиническим симптомом ХЭ было «неудачные попытки ЭКО» (таблица 2).

Так же стоит отметить, что ни у одной из пациенток не было моносимптоматического течения ХЭ. Как правило, полисимптоматичность формировалась из сочетания 3-х и более симптомов таких как неудачные попытки ЭКО, бесплодие с трубно-овариальными причинами, 
нарушение менструального цикла у 29 (48\%) пациенток обеих групп. Или неудачные попытки ЭКО, бесплодие с гормональными причинами, нарушение менструального цикла - у 21 (35\%) женщин. Воспалительные заболевания репродуктивной системы активно соседствовали с бесплодием (трубно-овариальные причины), нарушениями менструального цикла и неудачными попытками ЭКО - 19 (32\%) женщин.

Иммуногистохимическое исследование проводилось всем пациенткам двух групп до и после лечения. Полученные результаты показывают большую гетерогенность. Количество макрофагов CD68 после применения препарата уменьшается, но при этом макрофаги СО 163 демонстрируют обратную динамику. Плазмоциты CD138, несмотря на увеличение среднего числа и объемной плотности, демонстрируют выраженную негативную динамику. Количество клеток, экспрессирующих TOLL-подобные рецепторы, снижалось. Положительная динамика наблюдалась в 8 случаях (36\%), причем средний показатель прироста числа клеток составил $27 \%$. В оставшихся случаях (64\%) число клеток снижалось в среднем на 29,4\%. Положительная динамика наблюдалась в $13 \%$ случаях (59,5\%), средний показатель увеличения числа клеток - 39,4\% в остальных случаях - динамика отрицательная. Оценить динамику изменения количества плазмоцитов удалось не во всех случаях, потому что изначально в группе до лечения эти типы клеток отсутствовали. В 10 случаях СД 138 не было обнаружено вообще. После лечения появилась экспрессия плазмоцитов. Экспрессия TLR9 после применения препарата уменьшилась в 16 случаях (73\%), в остальных случаях количество клеток в среднем увеличилось на 66,5\%. Таким образом, на фоне приема препарата Деринат ${ }^{\circledR}$ существенно увеличилась количество клеток, участвующих в фагоцитарной активности макрофагов и клеточной пролиферации. После лечения у всех паци- енток была получена адекватная дню цикла гистологическая картина эндометрия и отсутствие воспалительных изменений.

\section{Обсужмение результатов}

Отмечено, что в основе проведения комплексного патогенетически обоснованного лечения при ХЭ лежат два механизма. В основе первого, способствующего восстановлению нарушенной ангиоархитектоники эндометрия и уменьшению степени ишемии ткани - предотвращение образования в эндометрии воспалительного инфильтрата (уменьшение количества иммунокомпетентных клеток (CD8, CD56, CD68, CD138), уровня провоспалительных цитокинов и факторов роста (IL-1, IL-6, IFN-y, TNF, TGF, VEGF и др.). Вследствие этого наблюдается восстановление. Ко второму противовоспалительному механизму относится нормализация регенеративных процессов в эндометрии на фоне снижения интенсивности апоптоза клеток и склеротических изменений в ткани, о чем свидетельствует восстановление показателей Ki-67, EGF, Apo-protein, уровней MMPs и различных видов коллагена. Важнейшим итогом комплексного лечения ХЭ является восстановление рецептивности эндометрия, что лежит в основе возможности успешной реализации репродуктивной функции в дальнейшем.

\section{Выво $\triangle \mathrm{b}$}

Результаты проведенного исследования свидетельствуют о целесообразности включения в комплексную терапию хронического эндометрита препарата деринат $^{\circledast}$, который обеспечивает лечению длительный положительный эффект, восстанавливает основные звенья иммунитета - все это позволяет улучшить репродуктивную функцию пациенток, у которых ранее ЭКО было неэффективно.

\section{ЛИТЕРАТУРА}

1. Дюжева Е. В. Гормональная подготовка эндометрия у пациенток с неэффективными попытками ЭКО в анамнезе.— М., 2010; дисс., канд. мед. наук.

2. Шишканова 0.Л. Оптимизация тактики лечения хронического эндометрита у пациенток с нарушением репродуктивной функции с использованием импульсной электротерапии. - М., 2008; дисс., канд. мед. наук.

3. Amui J., Check J. H., Cohen R. Successful twin pregnancy in a donor oocyte recipient despite a maximum endometrial thickness in the late proliferative phase of 4 mm // Clin. Exp. Obstet. Gyneco1. — 2011. — Vol. 38(4).— P. 328-329.

4. Kasius J.C., Broekmans F. J., Sie-Go Д.M., et a1. The reliability or the histological diagnosis or endometritis in asymptomatic IVF cases: a multicenter observer study // Hum. Reprod. - 2012.-Vol. 27.- P. 153-158.

5. Kasius J.C., Fatemi H. M., Bourgain C., et a1. The impact of chronic endometritis on reproductive outcome // Fertil. and Steril. — 2011.— Vol. 96(6). — P. 14511456.

6. Рудакова Е.Б., Мозговой С. И., Пилипенко М. А., и др. Хронический эндометрит: от совершенствования диагностического подхода к оптимизации лечения // Лечащий врач.— 2008.— № 10.- С. 610.

7. Senturk L.M., Ere C. T. Thin endometrium in assisted reproductive technology // Curr. Opin.0bstet. Gynecol. — 2008. — Vol. 20(3). — P. $221-228$.

8. Soares S.R., Velasco J. A., Fernandez M., et a1. Clinical factors affecting endometrial receptiveness in oocyte donation cycles // Fertil. and Steril. - 2008. - Vol. 89.- P. 491-501. 
9. Боярский К.Ю., Гайдуков С. Н., Пальченко Н. А. Современный взгляд на проблему рецептивности и тонкого эндометрия в программах ВРТ: обзор литературы // Проблемы репродукции. - 2013. - Т. 19., № 4.- С. 51-60.

10. Reynolds K., Khoury J., Sosnowski J., et a1. Comparison of the effect of tamoxifen on endometrial thickness in women with thin endometrium ( $<7 \mathrm{~mm})$ undergoing ovulation induction with clomiphene citrate // Fertil. and Steril.— 2010.— Vol. 93(6). — P. 2091-2093.

11. Волкова Е.Ю., Силантьева Е. С., Серов В.Н., и др. Влияние физиотерапии на гемодинамику матки у женщин с нарушением репродуктивной функции и «тонким» эндометрием // Российский вестник акушера-гинеколога. - 2012. — № 3. - C. 50-54.

12. Check J.H., Cohen R. Live fetus following embryo transfer in a woman with diminished egg reserve whose maximal endometrium thickness was less than $4 \mathrm{~mm} / /$ C1in. Exp. Obstet. Gynecol.—2011.—Vol. 38. - P. 330-332.

13. Калинина Е.А., Дюжева Е. В. Применение пероральных контрацептивов в программе экстракорпорального оплодотворения // Акуш. и гин.— 2009. № 2.- C. 11-13.

14. Miwa I., Tamura H., Takasaki A., et a1. Pathophysiologic features of 'thin' endometrium // Fertil. and Steril.— 2009.—Vol. 91(4). — P. $998-1004$.

15. Takasaki A., Tamura H., Miwa l., et a1. Endometrial growth and uterine blood: a pilot study for improving endometrial thickness in the patients with a thin endometrium // Fertil. And Steril.— 2010.—Vol. 93(6).—P. 1851-1858.

16. Рудакова Е.Б., Лобода 0. А. Хронический эндометрит в аспекте результативности программ экстрстракорпорального оплодотворения // Лечащий врач. 2012. - № 11.- C. 22-24.

17. Casper R. F. It's time to pay attention to the endometrium // Fertil. And Steril. — 2011. — Vol. 96. — P. 519-521.

18. Dix E., Check J. H. Successful pregnancies following embryo transfer despite very thin late proliferative endometrium // C1in. Exp. Obstet. Gyneco1.— 2010. — Vol. 37.- P. 15-16.

19. Филатов 0.Ю., Кашаева О. В., Бугримов Д. Ю., и др. Морфофизиологические принципы иммунологического действия ДНК эукариот // Российский Иммунологический журнал.— 2013.- Т. 7., № 16.- С. 4.

20. Jimenez P.T., Schon S. B., Odem R. R., et a1. A retrospective crossectional study: fresh cycle endometrial thickness is a sensitivepredictor of anadequate endometrial thickness in frozen embryo transfer cycles // Reprod. Bio1ndocrinol.— 2013.— Vol. 10.—P. 35.

( ) Косых Екатерина Владимировна ( ekaterina1991kosykh@gmail.com ), Коротких Ирина Николаевна, Филин Андрей Анатольевич.

Журнал «Современная наука: актуальные проблемы теории и практики»

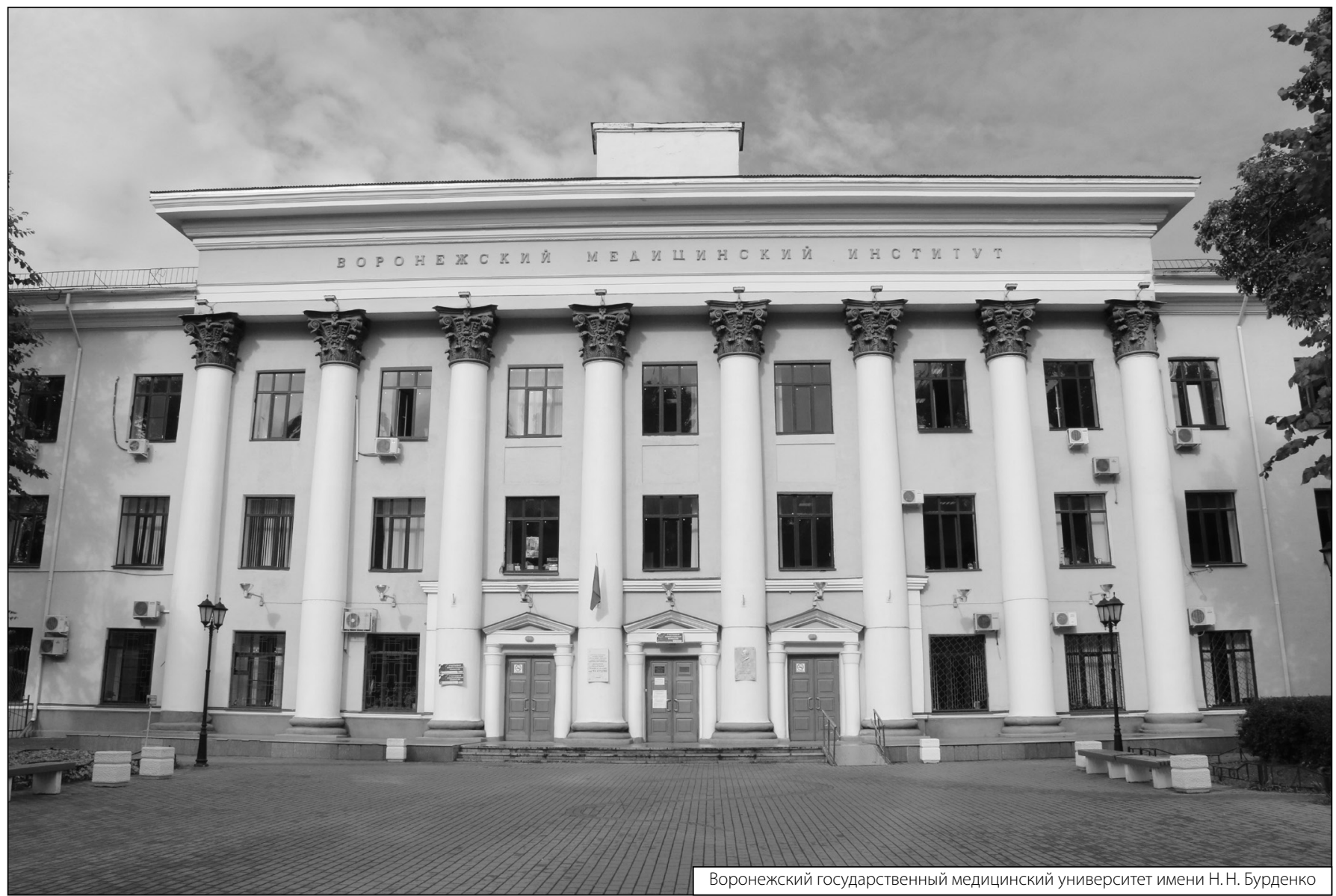

